\title{
The Error Estimates of Direct Discontinuous Galerkin Methods Based on Upwind-Baised Fluxes
}

\author{
Hui Bi*, Yixin Chen \\ College of Science, Harbin University of Science and Technology, Harbin, China \\ Email: ${ }^{\star 837610597 @ q q . c o m ~}$
}

How to cite this paper: $\mathrm{Bi}, \mathrm{H}$. and Chen, Y.X. (2020) The Error Estimates of Direct Discontinuous Galerkin Methods Based on Upwind-Baised Fluxes. Journal of Applied Mathematics and Physics, 8, 2964-2970. https://doi.org/10.4236/jamp.2020.812219

Received: November 9, 2020

Accepted: December 19, 2020

Published: December 22, 2020

\begin{abstract}
In this paper, we study the error estimates for direct discontinuous Galerkin methods based on the upwind-biased fluxes. We use a newly global projection to obtain the optimal error estimates. The numerical experiments imply that $L^{2}$ norms error estimates can reach to order $k+1$ by using time discretization methods.
\end{abstract}

\section{Keywords}

Direct Discontinuous Galerkin Methods, Global Projection, Error Estimates, The Upwind-Biased Fluxes

\section{Introduction}

The discontinuous Galerkin (DG) method was first proposed by Reed and Hill [1] to solve the neutron problems in 1973. With the development of DG, the direct discontinuous Galerkin (DDG) method [2] was proposed by Liu to solve the second order partial differential equations. The main idea of DDG is to direct force solve the higher order equation so as to avoid the reduction of the equation.

For the error analysis of DDG, we first got the linear result of the error estimates can reach to order $k$ in [3]. In [4], a series of special precision analyses were made for the numerical solution by using Fourier transform. The error estimates obtained by Liu can reach to order $k+1$ for the linear and nonlinear convection diffusion equations by using the DDG method in [5]. In 2016, Cao [6] discussed the superconvergence of DDG method and obtained that the projection superconvergence at some points can achieve order $k+2$. 
In this article, we use first order numerical fluxes to the diffusion term and use the upwind-biased fluxes to the convective term. The upwind-biased fluxes was first proposed by Meng and Shu, they proved that the optimal error estimates of the linear hyperbolic conservation equations can obtain order $k+1$ in semi-discrete and fully-discrete scheme in 2016 [7]. Meng extended the upwind-biased fluxes to the generalized alternating fluxes in [8].

The main content of this paper: In Section 2, we introduce the semi-discrete scheme of second-order partial differential equation and solve the error estimates problems by using the upwind-biased fluxes and first order numerical fluxes. In Section 3, we use the third-order RK time discretization methods for completing numerical experiments and obtain that the error estimates can reach order $k+1$.

\section{The Method of DDG}

This paper considers the following convection diffusion equation

$$
\begin{aligned}
& u_{t}+u_{x}=u_{x x}, \quad(x, t) \in[0,2 \pi] \times(0, T], \\
& u(x, 0)=u_{0}(x), \quad x \in R .
\end{aligned}
$$

For the convenience, we take the periodic boundary condition $u(0, t)=u(2 \pi, t)$ into discussion.

\subsection{The Meshes of DDG}

Let us denote the computational interval $I=[0,2 \pi]$, consisting of cells $I_{j}=\left(x_{j-1 / 2}, x_{j+1 / 2}\right)$, where $0=x_{1 / 2}<x_{3 / 2}<\cdots<x_{N+1 / 2}=2 \pi$.

We define $x_{j}=\left(x_{j-1 / 2}+x_{j+1 / 2}\right) / 2$ and $h=x_{j+1 / 2}-x_{j-1 / 2}$, and then use $x_{j+1 / 2}^{-}$ and $x_{j+1 / 2}^{+}$to denote the left and right limits at the discontinuity point. In what follows, we define $[x]=x^{+}-x^{-}$and $\{x\}=\left(x^{+}+x^{-}\right) / 2$. The following piecewise polynomials space is chosen as the finite element space

$$
V_{h} \equiv V_{h}^{k}=\left\{v \in L^{2}(I):\left.v\right|_{I_{j}} \in P^{k}\left(I_{j}\right), j=1, \cdots, N\right\},
$$

where $P^{k}\left(I_{j}\right)$ denotes the polynomials of degree up to $k \geq 0$ defined on cell $I_{j}$.

\subsection{Function Spaces and Norms}

Define the broken Sobolev spaces as

$$
W^{l, p}\left(I_{h}\right)=\left\{u \in L^{2}(I):\left.u\right|_{I_{j}} \in W^{l, p}\left(I_{j}\right), j=1, \cdots, N\right\}
$$

The norms of the broken Sobolev spaces with $p=2, \infty$ are given by: $\|u\|_{W^{l, 2\left(I_{j}\right)}}=\|u\|_{H^{l}\left(I_{j}\right)}=\left(\sum_{j=1}^{N}\|u\|_{H^{l}\left(I_{j}\right)}^{2}\right)^{\frac{1}{2}}$ and $\|u\|_{W^{l, \infty}\left(I_{j}\right)}=\max _{1 \leq j \leq N}\|u\|_{W^{l, \infty}\left(I_{j}\right)}$.

In the case $l=0$, we have $\|u\|_{L^{2}\left(I_{j}\right)}=\|u\|_{H^{0}\left(I_{j}\right)}$.

\subsection{The Semi-Discrete DDG Scheme}

The DDG scheme is defined as follows: find both $u_{h}$ and $v_{h}$ in $V_{h}^{k}$, by inte- 
gration by parts and need some interface corrections, the Equations (1) can be written as

$$
\begin{aligned}
& \int_{I_{j}}\left(u_{h}\right)_{t} v_{h} d x+\left.\widehat{u_{h}} v_{h}\right|_{j+\frac{1}{2}}-\left.\widehat{u_{h} v_{h}}\right|_{j-\frac{1}{2}}-\int_{I_{j}} u_{h}\left(v_{h}\right)_{x} d x-\left.\left(u_{h}\right)_{x} v_{h}\right|_{j+\frac{1}{2}} \\
& +\left.\left(u_{h}\right)_{x} v_{h}\right|_{j-\frac{1}{2}}+\int_{I_{j}}\left(u_{h}\right)_{x}\left(v_{h}\right)_{x} d x+\left.\frac{1}{2}\left[u_{h}\right]\left(v_{x}\right)^{-}\right|_{j+\frac{1}{2}}+\left.\frac{1}{2}\left[u_{h}\right]\left(v_{x}\right)^{+}\right|_{j-\frac{1}{2}}=0, \\
& \int_{I_{j}} u_{h}(x, 0) v_{h} d x=\int_{I_{j}} u_{0} v_{h} d x,
\end{aligned}
$$

Summing $j$ we have

$$
\begin{aligned}
& \sum_{j=1}^{N} \int_{I_{j}}\left(u_{h}\right)_{t} v_{h} d x+\sum_{j=1}^{N}\left(-\int_{I_{j}} u_{h}\left(v_{h}\right)_{x} d x+\int_{I_{j}}\left(u_{h}\right)_{x}\left(v_{h}\right)_{x} d x\right) \\
& +\sum_{j=1}^{N}\left(\left(-\widehat{u_{h}}+\left(\widehat{\left.u_{h}\right)_{x}}\right)\left[v_{h}\right]+\left(\widehat{\left.v_{h}\right)_{x}}\left[u_{h}\right]\right)_{j+1 / 2}=0 .\right.\right.
\end{aligned}
$$

Here $\widehat{u_{h}}$ is the upwind-biased fluxes as: $\widehat{u_{h}}=u_{h}^{\theta}=\theta u_{h}^{-}+(1-\theta) u_{h}^{+}$, where $\theta>\frac{1}{2}$.

Following [2] we take

$$
\widehat{\left(u_{h}\right)_{x}}=\frac{\beta_{0}}{h}\left[u_{h}\right]+\left\{\left(u_{h}\right)_{x}\right\}+\beta_{1} h\left[\left(u_{h}\right)_{x x}\right], \quad \widehat{\left(v_{h}\right)_{x}}=\left\{\left(v_{h}\right)_{x}\right\} .
$$

We define two operators

$$
\begin{gathered}
A\left(u_{h}, v_{h}\right)=\sum_{j=1}^{N} \int_{I_{j}}\left(u_{h}\right)_{x}\left(v_{h}\right)_{x} d x+\sum_{j=1}^{N}\left(\widehat{\left(u_{h}\right)_{x}}\left[v_{h}\right]+\left[u_{h}\right]\left\{\left(v_{h}\right)_{x}\right\}\right)_{j+\frac{1}{2}}, \\
F\left(u_{h}, v_{h}\right)=\sum_{j=1}^{N} u_{h}\left(v_{h}\right)_{x} d x+\left(\sum_{j=1}^{N} \widehat{u_{h}}\left[v_{h}\right]\right)_{j+\frac{1}{2}} .
\end{gathered}
$$

So the Equation (3) can be written as

$$
\left\langle\left(u_{h}\right)_{t}, v_{h}\right\rangle+A\left(u_{h}, v_{h}\right)=F\left(u_{h}, v_{h}\right), \quad \forall v_{h} \in V_{h}^{k} .
$$

We define energy norm and introduce a quantity

$$
\begin{gathered}
\|v\|_{E}^{2}=\sum_{j=1}^{N} \int_{I_{j}}\left|v_{x}\right|^{2} d x+\sum_{j=1}^{N} \frac{\beta_{0}}{h}[v]_{j+\frac{1}{2}}^{2}, \quad v \in V_{h}^{k}, \\
\Gamma\left(\beta_{1}\right)=\sup _{v \in P^{k-1}[-1,1]} \frac{\left(v(1)-2 \beta_{1} \partial_{\xi} v(1)\right)^{2}}{f_{-1}^{1} v^{2}(\xi) d \xi},
\end{gathered}
$$

where $\xi=2\left(x-x_{j}\right) / h$ and $\frac{\int_{u^{-}}^{u^{+}} f(u) d u}{[u]}=f_{u^{-}}^{u^{+}} u d u$.

According to [3] there exists $\gamma \in(0,1)$ such that

$$
A(v, v) \geq \gamma\|v\|_{E}^{2}, \quad \forall v \in V_{h}^{k},
$$

and $\beta_{0}>\Gamma\left(\beta_{1}\right)$.

Lemma 1 For a quadratic entropy flux, it holds that

$$
F\left(u_{h}, u_{h}\right) \leq 0 .
$$


Proof

A quadratic entropy flux satisfies [9]

$$
\int_{u^{-}}^{u^{+}}\left(\hat{f}\left(u^{+}, u^{-}\right)-f(u)\right) d u=\left(\hat{f}\left(u^{+}, u^{-}\right)-\frac{\int_{u^{-}}^{u^{+}} f(u) d u}{[u]}\right)[u] \leq 0,
$$

Firstly we figure out that $\sum_{j=1}^{N} \int_{I_{j}} u_{h}\left(u_{h}\right)_{x} d x=-\sum_{j=1}^{N}\left(\left(f_{u^{-}}^{u^{+}} u_{h} d u_{h}\right)\left[u_{h}\right]\right)_{j+1 / 2}$. Then using Equation (11) we get

$$
\begin{aligned}
F\left(u_{h}, u_{h}\right) & =\sum_{j=1}^{N}\left(\widehat{u_{h}}\left[u_{h}\right]\right)_{j+1 / 2}-\sum_{j=1}^{N}\left(\left(f_{u^{-}}^{u^{+}} u_{h} d u_{h}\right)\left[u_{h}\right]\right)_{j+1 / 2} \\
& =-\sum_{j=1}^{N}\left(\widehat{u_{h}}-\frac{\int_{u^{-}}^{u^{+}} u_{h} d u_{h}}{\left[u_{h}\right]}\right)\left[u_{h}\right]_{j+1 / 2} \leq 0 .
\end{aligned}
$$

\subsection{The Stability of DDG}

Theorem 1 Consider the semi-discrete of DDG, it satisfies the following properties:

1) Conservation of mass: $\sum_{j=1}^{N} \int_{I_{j}} u_{h}(t, x) d x=\int_{I} u_{0}(x) d x, \forall t>0$.

2) There exists $\gamma \in(0,1)$ such that

$$
\frac{d}{d t}\left\|u_{h}\right\|^{2} \leq-2 \gamma\left\|u_{h}\right\|_{E}^{2} \leq 0
$$

3) The scheme is $L^{2}$ stable: $\left\|u_{h}\right\|^{2} \leq \int_{I} u_{0}^{2} d x, \forall t>0$.

Proof

1) Taking $v_{h}=1$ into Equation (6) we have $\frac{d}{d t} \sum_{j=1}^{N} \int_{I_{j}} u_{h} d x=0$. Combining with Equation (2) with $v_{h}=1$ leads to the mass conservation.

2) Taking $v_{h}=u_{h}$ into Equation (6), we obtain

$$
\frac{1}{2} \frac{d}{d t}\left\|u_{h}\right\|^{2}+A\left(u_{h}, u_{h}\right)=F\left(u_{h}, u_{h}\right)
$$

According Equation (9) and combining with Lemma 1 together prove the Equation (12).

3) It follows from Equations (12) and (2) that

$$
\left\|u_{h}\right\|^{2} \leq\left\|u_{h}(x, 0)\right\|^{2}=\sum_{j=1}^{N} \int_{I_{j}} u_{h}^{2}(x, 0) d x \leq \sum_{j=1}^{N} \int_{I_{j}} u_{0}^{2} d x .
$$

\subsection{The Global Projections}

For the DDG method using the upwind-biased fluxes, we need to construct a globally projection $P$. For $u \in H^{1}(I)$, the projection $P$ is defined as

$$
\begin{aligned}
& \int_{I_{j}}(P u-u) v d x=0, \quad \forall v \in P^{k-2}\left(I_{j}\right), j=1, \ldots, N, \\
& (\widehat{P u})_{x}-\hat{u}=\widehat{u_{x}}-u, \\
& \{P u\}=\{u\},
\end{aligned}
$$


with $\theta>\frac{1}{2}$.

We quote the lemma as follows [5]

Lemma 2 For $\theta>\frac{1}{2}$ and $\beta_{0}>\Gamma\left(\beta_{1}\right)$, the projection $P$ holds that

$$
\|u-P u\|+h\|u-P u\|_{\infty}+h^{1 / 2}\|u-P u\|_{\Gamma_{h}} \leq C h^{k+1},
$$

where $C$ is independent of $h$ and depends on $\theta, \beta_{0}, \beta_{1}$.

\subsection{The Error Estimates of DDG}

Theorem 1 Assume that $u$ are the exact solutions, we take the upwind-baised fluxes and the finite element space $V_{h}^{k}$, there hold the following error estimates

$$
\left\|u-u_{h}\right\|_{L^{2}(I)} \leq C h^{k+1},
$$

where $C$ is independent of $h$ and depends on $\theta, \beta_{0}, \beta_{1}$.

Proof

Firstly we set

$$
e=P u-u_{h}, \quad \epsilon=P u-u,
$$

Since both the exact and numerical solutions satisfy the weak solution form, we have

$$
\left(e_{t}, e\right)+A(e, e)-F(e, e)=\left(\epsilon_{t}, e\right)+A(\epsilon, e)-F(\epsilon, e) .
$$

For the left side we use $F(e, e) \leq 0$ to obtain

$$
\left(e_{t}, e\right)+A(e, e)-F(e, e) \geq \frac{1}{2} \frac{d}{d t} \int_{0}^{1} e^{2} d x+\gamma\|e\|_{E}^{2} .
$$

And for the right side using the definition of projection we have

$$
\begin{aligned}
A(\epsilon, e) & =\sum_{j=1}^{N} \int_{I_{j}} \epsilon_{x} e_{x} d x+\sum_{j=1}^{N}\left(\widehat{\epsilon_{x}}[e]+[\epsilon]\left\{e_{x}\right\}\right)_{j+\frac{1}{2}} \\
& =-\sum_{j=1}^{N} \int_{I_{j}} e_{x x} \epsilon d x+\sum_{j=1}^{N}\left(\widehat{\epsilon_{x}}[e]-\{\epsilon\}\left[e_{x}\right]\right)_{j+\frac{1}{2}},
\end{aligned}
$$

Thus, we get

$$
F(\epsilon, e)=\sum_{j=1}^{N} \epsilon e_{\chi} d x+\left(\sum_{j=1}^{N} \hat{\epsilon}[e]\right)_{j+\frac{1}{2}} .
$$

Summing $A(\epsilon, e)$ and $F(\epsilon, e)$ to obtain

$$
\left.A(\epsilon, e)-F(\epsilon, e)=-\sum_{j=1}^{N} \int_{I_{j}}\left(e_{x x} \epsilon+\epsilon e_{\chi}\right) d x+\sum_{j=1}^{N}\left(\hat{\left(\epsilon_{x}\right.}-\epsilon\right)[e]-\{\epsilon\}\left[e_{x}\right]\right)_{j+\frac{1}{2}},
$$

According Equation (13) the highest order is $k-2$. We have $\int_{I_{j}} e_{x x} \epsilon d x=0$. And by the properties of projection we obtain

$$
\widehat{\epsilon_{x}}=(P u)_{x}-u_{x}=0, \quad\{\epsilon\}=\{P u\}-\{u\}=0 .
$$

So the right side of Equation (16) can be written as

$$
\left(\epsilon_{t}, e\right)+A(\epsilon, e)-F(\epsilon, e) \leq \frac{1}{2} \int_{I_{j}} \epsilon_{t}^{2} d x+\int_{I_{j}} e^{2} d x+\frac{\alpha^{2}}{2 \gamma} \int_{I_{j}} \epsilon^{2} d x+\frac{\gamma}{2}\|e\|_{E}^{2} .
$$


Combining Equation (17), Equation (21) and Lemma 2, we have

$$
\frac{d}{d t} \int_{I_{j}} e^{2} d x+\gamma\|e\|_{E}^{2} \leq \int_{I_{j}} e^{2} d x+C h^{2 k+2} .
$$

Finally by using the Gronwall inequality, we obtain Theorem 1.

\section{Numerical Experiments}

We present numerical experiments to validate the error estimates of DDG method based on upwind-biased fluxes. We adopt $P^{k}$ elements on the uniform mesh, with $N=10,20,40,80$. In order to reduce time errors, we use the third order Runge-Kutta method and compute until $T=1$.

For time discretization, we use TVD type third-order Runge-Kutta method [10]

$$
\begin{aligned}
& u^{(1)}=u^{n}+\Delta t R\left(u^{n}, t\right), \\
& u^{(2)}=\frac{3}{4} u^{n}+\frac{1}{4}\left[u^{(1)}+\Delta t R\left(u^{(1)}, t+\Delta t\right)\right], \\
& u_{n+1}=\frac{1}{3} u^{n}+\frac{2}{3}\left[u^{(2)}+\Delta t R\left(u^{(2)}, t+0.5 \Delta t\right)\right] .
\end{aligned}
$$

Consider the equation

$$
\begin{aligned}
& u_{t}+u_{x}=u_{x x}, \\
& u(x, 0)=u_{0}(x)=\sin x .
\end{aligned}
$$

The exact solution of the equation is $u(x, t)=e^{-t} \sin (x-t)$.

Table 1 shows that the error estimates of the convection diffusion equation by using the DDG method and the upwind-biased fluxes can reach to the order $k+1$, With the coefficients $\theta$ changes, the results change together, so we can choose the best error results.

Table 1. The $L^{2}$ error estimates until $T=1$.

\begin{tabular}{cccccccccc}
\hline$k=0$ & \multicolumn{2}{c}{$\theta=0.5$} & \multicolumn{2}{c}{$\theta=0.8$} & \multicolumn{2}{c}{$\theta=1$} & \multicolumn{2}{c}{$\theta=1.5$} \\
\hline$N$ & error & order & error & order & error & order & error & order \\
\hline 10 & $1.16 \mathrm{E}-01$ & - & $1.24 \mathrm{E}-01$ & - & $1.32 \mathrm{E}-01$ & - & $1.61 \mathrm{E}-01$ & - \\
20 & $5.58 \mathrm{E}-02$ & 1.05 & $5.90 \mathrm{E}-02$ & 1.07 & $6.29 \mathrm{E}-02$ & 1.07 & $7.74 \mathrm{E}-02$ & 1.05 \\
40 & $2.75 \mathrm{E}-02$ & 1.02 & $2.88 \mathrm{E}-02$ & 1.04 & $3.06 \mathrm{E}-02$ & 1.04 & $3.79 \mathrm{E}-02$ & 1.03 \\
80 & $1.36 \mathrm{E}-02$ & 1.01 & $1.42 \mathrm{E}-02$ & 1.02 & $1.51 \mathrm{E}-02$ & 1.02 & $1.87 \mathrm{E}-02$ & 1.02 \\
$k=1$ & $\theta=0.5$ & \multicolumn{1}{c}{$\theta=0.8$} & & $\theta=1$ & & $\theta=1.5$ & \\
$N$ & error & order & error & order & error & order & error & order \\
10 & $2.29 \mathrm{E}-02$ & - & $1.93 \mathrm{E}-02$ & - & $2.25 \mathrm{E}-02$ & - & $2.72 \mathrm{E}-02$ & - \\
20 & $6.23 \mathrm{E}-03$ & 1.86 & $5.93 \mathrm{E}-03$ & 1.70 & $6.56 \mathrm{E}-03$ & 1.78 & $7.41 \mathrm{E}-03$ & 1.88 \\
40 & $1.72 \mathrm{E}-03$ & 1.86 & $1.60 \mathrm{E}-03$ & 1.89 & $1.69 \mathrm{E}-03$ & 1.96 & $1.83 \mathrm{E}-03$ & 2.02 \\
80 & $4.74 \mathrm{E}-04$ & 1.86 & $4.36 \mathrm{E}-04$ & 1.88 & $4.54 \mathrm{E}-04$ & 1.90 & $4.85 \mathrm{E}-04$ & 1.92 \\
$k=2$ & $\theta=0.5$ & & $\theta=0.8$ & & $\theta=1$ & & $\theta=1.5$ & \\
$N$ & error & order & error & order & error & order & error & order \\
10 & $3.07 \mathrm{E}-03$ & - & $2.83 \mathrm{E}-03$ & - & $2.72 \mathrm{E}-03$ & - & $2.52 \mathrm{E}-03$ & - \\
20 & $3.77 \mathrm{E}-04$ & 3.02 & $3.63 \mathrm{E}-04$ & 2.96 & $3.55 \mathrm{E}-04$ & 2.94 & $3.38 \mathrm{E}-04$ & 2.90 \\
40 & $4.51 \mathrm{E}-05$ & 3.06 & $4.42 \mathrm{E}-05$ & 3.04 & $4.37 \mathrm{E}-04$ & 3.02 & $4.24 \mathrm{E}-04$ & 2.99 \\
80 & $4.55 \mathrm{E}-06$ & 3.31 & $4.49 \mathrm{E}-06$ & 3.30 & $4.56 \mathrm{E}-06$ & 3.26 & $4.48 \mathrm{E}-06$ & 3.24 \\
\hline
\end{tabular}




\section{Conclusion}

Based on the idea of DDG method and the upwind-biased fluxes, this paper proves the stability of numerical solutions and the error estimates of convection diffusion equation can reach to the order $k+1$. Numerical experiments show that the scheme is stability and the error estimates is accurate.

\section{Conflicts of Interest}

The authors declare no conflicts of interest regarding the publication of this paper.

\section{References}

[1] Reed, W.H. and Hill, T.R. (1973) Triangular Mesh Methods for the Neutron Transport Equation. Los Alamos Report LA-UR, 473-479.

[2] Liu, H. and Yan, J. (2008) The Direct Discontinuous Galerkin (DDG) Methods for Diffusion Problems. SIAM J. Numer. Anal, 567-698. https://doi.org/10.1137/080720255

[3] Yan, J. and Liu, H. (2010) The Direct Discontinuous Galerkin (DDG) Method for Diffusion with Interface Corrections. Commun. Comput. Phys, 541-564. https://doi.org/10.4208/cicp.010909.011209a

[4] Zhang, M. and Yan, J. (2012) Fourier Type Error Analysis of the Direct Discontinuous Galerkin Method and Its Variations for Diffusion Equations. J. Sci. Comput, 638-655. https://doi.org/10.1007/s10915-011-9564-5

[5] Liu, H. (2015) Optimal Error Estimate of DDG for Convection-Diffusion Equations. Math. Comp, 2263-2295. https://doi.org/10.1090/S0025-5718-2015-02923-8

[6] Cao, W., Liu, H. and Zhang, Z.M. (2017) Superconvergence of the Direct Discontinuous Galerkin Method for Convection-Diffusion Equations. Numerical Methods for Partial Differential Equations. https://doi.org/10.1002/num.22087

[7] Meng, X., Shu, C.W. and Wu, B. (2016) Optimal Error Estimates for Discontinuous Galerkin Methods Based on Upwind-Biased Fluxes for Linear Hyperbolic Equations. Math. Comp, 1225-1261. https://doi.org/10.1090/mcom/3022

[8] Cheng, Y., Meng, X. and Zhang, Q. (2016) Application of Generalized Gauss-Radau Projections for the Local Discontinuous Galerkin Method for Linear Convection-Diffusion Equations. Math.Comp, 1233-1267. https://doi.org/10.1090/mcom/3141

[9] Osher, S. and Riemann, S. (1984) The Entropy Condition and Difference Approximations. SIAM J. Numer. Anal, 217-235. https://doi.org/10.1137/0721016

[10] Zhang, Y.G. and Ma, Y.F. (2017) High Order Compact Difference Scheme for Solving the Unsteady Convection Diffusion Reaction Equation. Mathematics in Practice and Theory, 263-270. 